\title{
Promoting the understanding of sex differences to enhance equity and excellence in biomedical science
}

\author{
Arthur P Arnold
}

From the moment of our conception, each of us has a sex. Sex has a major role in determining the physical attributes of our bodies, the structure of our brains, our behavioral tendencies, our susceptibility and reaction to diseases, the environment in which we grow up, our place in society, the attitudes of others towards us, and our conception of self. Although sex may be considered to be determined primarily biologically, our gender (i.e., the social perception and implications of our sex) is arguably equally or more important for our lives. Sex and gender differences are created by an intricate reciprocal interaction of numerous biological and environmental forces.

Biology of Sex Differences publishes articles concerning the role of sex and gender in normal traits and disease. As the official journal of the Organization for the Study of Sex Differences (http://www.ossdweb.org), Biology of Sex Differences will serve as a forum for discussion of the forces that make females and males different, and the downstream pathways that are affected by sex-specific forces. Biology of Sex Differences will publish articles on animal models and humans. The launch of Biology of Sex Differences is the result of several major developments in biomedicine in recent years.

\section{Sex matters}

There is increasing evidence that males and females differ in their basic physiology, and in the susceptibility to and progression of diseases. The 2001 US Institute of Medicine report [1] concluded that sex matters in major diseases and response to therapy, and sometimes the effect of sex is profound. Women suffer from autoimmune diseases much more frequently than men. For example, women have a nine-fold greater incidence of systemic lupus erythematosus than men, and up to three times greater

Correspondence: arnold@ucla.edu

Department of Integrative Biology and Physiology, University of California, Los Angeles incidence of multiple sclerosis [2,3]. Males suffer more from some brain diseases such as attention deficit hyperactivity disorder, autism spectrum disorders, Tourette's syndrome, Parkinson's disease, etc. $[4,5]$. Sex differences occur in metabolic disease, hypertension, cardiovascular disease, cancer, and response to drugs [6-10]. In some cases, the sex differences are complex, changing over the lifespan or as a function of environment, suggesting that there are multiple sex-biased or sex-specific factors that need to be understood. At the very least, explaining the sex-specific causal variables should help physicians and patients anticipate important changes over the lifespan. More importantly, understanding these factors could uncover sex-specific protective forces that are attractive targets for novel therapies.

\section{Biomedical science has shown a strong sex bias and blindness to sex}

Analysis of the scientific literature in numerous disciplines leads to the general conclusion that males are studied much more than females [12], in both animal and human research, so that the basic biology of medical texts may better reflect the biology of males than females. Drug development is based on research on males, even for diseases that are more prominent in females, and despite evidence that drug metabolism and efficacy differ in the two sexes $[10,11]$. Moreover, the sex of the experimental subjects is often not recorded in scientific reports [12]. For this reason, sex differences in physiology and disease are unappreciated simply because we don't have the data to know when differences occur, how large they are, and what causes them. Importantly, however, some sexspecific factors counteract each other [13], and make the two sexes more equivalent in phenotype rather than causing differences. For example, inactivation of one of the two $\mathrm{X}$ chromosome in every non-germline cell makes $\mathrm{XX}$ female cells more similar to XY male cells, in gene expression and function $[14,15]$. Thus, appreciating the impact 
of sex on cell function requires not just studying functions that are overtly different in the two sexes, but also those that are equivalent because of sex-specific factors that cancel each other out.

\section{The study of sex differences is a field of its own}

Historically, sex differences have been studied most often in tissues involved in reproduction, where the differences are large: the gonads, internal and external genitalia, and brain and behavior. In the last century, these studies have given rise to general theories of sexual differentiation, about the genetic origins of sex differences, and the sex-specific signals that drive sexual differentiation of many tissues [16]. These studies result in a set of attitudes, animal models, and experimental protocols that can be applied to the study of sex differences of any tissue $[17,18]$. This common framework for studying sex differences is continuously evolving as it is broadened and applied to different research problems. Biology of Sex Differences will serve as a platform for ideas contributing to the evolution of the discipline.

Sex differences in physiology and disease will be front and center in Biology of Sex Differences, not a sub-topic. Unlike journals that focus on specific physiological systems or diseases, Biology of Sex Differences will aim to compare sex-specific effects across tissues, species, and diseases. The goal is to build on knowledge of sex differences in various systems to understand more fully how sex differences impact the entire organism.

\section{New understanding of the biology of sex differences}

The revolution in molecular biology and genetics is rapidly advancing our understanding of the sex chromosomes, which are the only factors (in animals with heteromorphic sex chromosomes) that are thought to differ consistently in female and male zygotes, and which therefore give rise to all subsequent sex differences $[16,19]$. Understanding sex differences in phenotype and disease must therefore be related eventually to some fundamental differences in these chromosomes, which cause a constitutive sexual inequality in the expression of $\mathrm{X}$ or $\mathrm{Y}$ genes. Multiple $\mathrm{X}$ and $\mathrm{Y}$ genes cause sex differences, but most of them have not been identified $[16,20]$. Of primary importance in mammals is the $\mathrm{Y}$ chromosome gene Sry, which initiates molecular and cellular cascades in the undifferentiated gonads of males that lead to differentiation of testes [21]. In the female gonads, other pathways are activated that cause ovarian differentiation and suppress testicular development [22]. The sexual differentiation of the gonads leads to lifelong differences in the levels of gonadal hormones, which cause many or most sex differences in other tissues $[16,23]$. The downstream pathways responding to sex-specific hormonal and genetic signals are complex, tissue- and age-specific, and poorly understood. Biology of Sex Differences will promote understanding of the sex-specific signals and their downstream pathways and effects, including their impact on disease processes.

\section{"Nothing in biology makes sense except in light of evolution"}

Dobzhansky's [24] famous admonition holds true for the study of sex differences. The evolution of the mammalian genome only makes sense in light of the differences in the sex chromosomes, the differential fitness of alleles in the two sexes, and the co-evolution of molecular pathways in the two sexes. The evolution of the sex chromosomes, for example, is thought to set up contrasting selection pressures in the two sexes, so that understanding the organization of modern genomes requires an evolutionary perspective [25]. In mammals, some sex-specific forces in females can be understood as a response to the constraint of having two $\mathrm{X}$ chromosomes and ovaries, and other forces in males are likely to be an evolutionary response to having a single X, Sry and testes. Each sex is constrained into specific adaptations because of previously existing sex differences in the genome, physiology, and ecological niche. The study of evolution must be a central part of the biology of sex differences.

We can only appreciate sex differences in physiology and disease by a broad study of numerous animal models, because important principles are more obvious in some species than others. Not infrequently, study of non-mammalian model systems has revealed principles that were later found also to be true for mammals including humans, but were not as easily discovered in mammals [26].

\section{The importance of sex-specific environments}

It is abundantly clear, from research and casual observation, that girls and boys are reared in fundamentally different environments, and schooled within a highly gendered framework of expectations. Because sex-specific environmental forces correlate so strongly with sexspecific biological factors, it is often impossible to separate their effects, especially in humans. Environmental factors causing sex differences are often routinely excluded or ignored by biologists because they have been considered to be outside of the realm of biology. Nevertheless, such forces interact strongly with biological factors. It is time to be inclusive, and purposely expand the biological investigation of sex differences to incorporate the interaction of environmental and biological factors. Especially now, as the epigenetic effects of environments on biological function are just beginning to be appreciated at the molecular level [27-29], the study of sex-specific environments must be incorporated into the biological study of sex differences. 


\section{Studying sex differences is an important strategy for understanding diseases and for developing therapies}

The existence of sex differences in disease implies that one sex has a sex-specific factor or process that protects from the disease. If that factor can be enhanced or modified, by direct manipulation of the factor or its downstream pathways, then the effects of the disease may be mitigated in both sexes. Because most factors causing sex differences are not lethal, they are attractive targets for drug development. The current lack of understanding of the biological basis of sex differences in disease may represent a large untapped reservoir of information relevant to development of novel therapies.

\section{Equality and Diversity in Clinical Practice}

Biology of Sex Differences will serve as a forum for translating basic science into clinical practice. We welcome articles studying basic biology, that translate basic biology into medical practice, and that measure sex differences in the clinic and in the incidence and progression of human disease.

Sex and age are the two main modifiers of disease risk, presentation, and treatment. Treating one sex like the other may be as inappropriate as treating a child like an adult. To improve health for both women and men, we must understand specifically how sex modifies disease risk, presentation, and treatment. The equitable treatment of females and males requires an understanding of their differences, which provides a necessary foundation for an appropriate response to their individual differences. To ignore the differences is as dangerous as it is to overemphasize them. A goal of the Biology of Sex Differences is to increase understanding of the sex-specific forces that make the sexes different, and make them more similar than they otherwise would be.

\section{Acknowledgements}

Thanks to Jill Becker, Sarah Berga, Carolyn Brown, Geert De Vries, Anne Etgen, Virginia Miller, and Viviana Simon, who made numerous suggestions that improved a draft of this editorial.

Received: 3 November 2010 Accepted: 4 November 2010 Published: 4 November 2010

\section{References}

1. US National Institute of Medicine Committee on Understanding the Biology of Sex and Gender Disorders: Exploring the Biological Contributions to Human Health: Does Sex Matter? Washington D.C.: National Academy Press; 2001.

2. Whitacre CC, Reingold SC, O'Looney PA: A gender gap in autoimmunity. Science 1999, 283:1277-1278.

3. Voskuhl RR: Sex differences in autoimmune diseases. In Hormones, Brain, and Behavior. Edited by: Pfaff DW, Arnold AP, Etgen AM, Fahrbach SE, Rubin RT. Elsevier; 2009:2259-2290.

4. Swaab DF, Hofman MA: Sexual differentiation of the human hypothalamus in relation to gender and sexual orientation. Trends Neurosci 1995, 18:264-270.
5. Zup SL, Forger NG: Hormones and sexual differentiation. In In Encyclopedia of the Human Brain. Edited by: Ramachandran VS. San Diego: Academic Press; 2002:323-341.

6. Luczak ED, Leinwand LA: Sex-based cardiac physiology. Annu Rev Physiol 2009, 71:1-18.

7. Lovejoy JC, Sainsbury A: Sex differences in obesity and the regulation of energy homeostasis. Obes Rev 2009, 10:154-167.

8. Lopez-Ruiz A, Sartori-Valinotti J, Yanes LL, lliescu R, Reckelhoff JF: Sex differences in control of blood pressure: role of oxidative stress in hypertension in females. Am J Physiol Heart Circ Physiol 2008, 295: H466-H474.

9. Paggi MG, Vona R, Abbruzzese C, Malorni W: Gender-related disparities in non-small cell lung cancer. Cancer Lett 2010, 298:1-8.

10. Nicolson TJ, Mellor HR, Roberts RR: Gender differences in drug toxicity. Trends Pharmacol Sci 2010, 31:108-114.

11. Franconi F, Brunelleschi S, Steardo L, Cuomo V: Gender differences in drug responses. Pharmacol Res 2007, 55:81-95.

12. Beery AK, Zucker I: Sex bias in neuroscience and biomedical research. Neurosci Biobehav Rev 2010

13. De Vries GJ: Minireview: Sex differences in adult and developing brains: compensation, compensation, compensation. Endocrinol 2004, 145:1063-1068.

14. Chow JC, Yen Z, Ziesche SM, Brown CJ: Silencing of the mammalian X chromosome. Annu Rev Genomics Hum Genet 2005, 6:69-92.

15. Nguyen DK, Disteche CM: Dosage compensation of the active $X$ chromosome in mammals. Nat Genet 2006, 38:47-53.

16. Arnold AP: The organizational-activational hypothesis as the foundation for a unified theory of sexual differentiation of all mammalian tissues. Horm Behav 2009, 55:570-578.

17. Becker JB, Arnold AP, Berkley KJ, Blaustein JD, Eckel LA, Hampson E, Hampson E, Herman JP, Marts S, Sadee W, Steiner M, Taylor J, Young E: Strategies and methods for research on sex differences in brain and behavior. Endocrinol 2005, 146:1650-1673.

18. Greenspan JD, Craft RM, LeResche L, rendt-Nielsen L, Berkley KJ, Fillingim RB, Goldh MS, Holdcrofti A, Lautenbacherj S, Mayerk EA, Mogill JS, Murphym AZ, Traubab RJ: Studying sex and gender differences in pain and analgesia: a consensus report. Pain 2007, 132(Suppl 1):S26-S45.

19. Arnold AP: Sex chromosomes and brain gender. Nat Rev Neurosci 2004, 5:701-708.

20. Arnold AP: Mouse models for evaluating sex chromosome effects that cause sex differences in non-gonadal tissues. J Neuroendocrinol 2009, 21:377-386.

21. Tilmann C, Capel B: Cellular and molecular pathways regulating mammalian sex determination. Recent Prog Horm Res 2002, 57:1-18.

22. Koopman P: The delicate balance between male and female sex determining pathways: potential for disruption of early steps in sexual development. Int J Androl 2010, 33:252-258.

23. van NA, GuhaThakurta D, Wang SS, Yehya N, Horvath S, Zhang B, IngramDrake L, Chaudhuri G, Schadt EE, Drake TA, Arnold AP, Lusis AJ: Elucidating the role of gonadal hormones in sexually dimorphic gene coexpression networks. Endocrinol 2009, 150:1235-1249.

24. Dobzhansky T: Nothing in biology makes sense except in light of evolutoin. American Biology Teacher 1973, 35:125-129.

25. Graves JAM: Sex chromosome specialization and degeneration in mammals. Cell 2006, 124:901-914.

26. Nottebohm F, editor: Hope for a new neurology New York: NY Academy of Sciences; 1985

27. Szyf M: The early life environment and the epigenome. Biochim Biophys Acta 2009, 1790:878-885.

28. Turner BM: Epigenetic responses to environmental change and their evolutionary implications. Philos Trans $R$ Soc Lond B Biol Sci 2009, 364:3403-3418.

29. Nadeau $\mathrm{JH}$ : Transgenerational genetic effects on phenotypic variation and disease risk. Hum Mol Genet 2009, 18:R202-R210.

doi:10.1186/2042-6410-1-1

Cite this article as: Arnold: Promoting the understanding of sex differences to enhance equity and excellence in biomedical science. Biology of Sex Differences 2010 1:1. 